\title{
Lifetime prevalence
}

INSERM

\section{Source}

INSERM. (1999). Orphanet: an online rare disease and orphan drug data base. lifetime prevalence. ORPHA:409969

Number of cases presenting or having presented the clinical entity during their lifetime scaled up to the general population. 